\title{
Lattice dynamics of cryocrystals at high pressure
}

\author{
E.E. Horbenko ${ }^{1,2}$, E.P. Troitskaya ${ }^{1}$, and Val.V. Chabanenko ${ }^{1}$ \\ ${ }^{1}$ Donetsk A.A. Galkin Institute of Physics and Technology of the \\ National Academy of Sciences of Ukraine, 72 R. Luxemburg Str., Donetsk 83114, Ukraine \\ ${ }^{2}$ Luhansk Taras Shevchenko National Pedagogical University, 2 Oboronna Str., Luhansk 91011, Ukraine \\ E-mail: e_g81@mail.ru
}

Received October 20, 2006

\begin{abstract}
The lattice dynamics of cryocrystals is investigated from first principles in the framework of the Tolpygo model over a wide range of pressures. The phonon frequencies in rare-gas solids are calculated in terms of models that go beyond the scope of adiabatic approximation. At high pressure the phonon spectrum along the $\Delta$ and $\Sigma$ directions is distorted and the longitudinal $L$ - and transverse $T_{2}$-modes soften as a result of the electron-phonon interaction, with the relative contribution decreasing in the sequence Ar, Kr, Xe. The calculated phonon frequencies are in good agreement with the experimental data for argon crystals of a pressure $3.1 \mathrm{GPa}$.
\end{abstract}

PACS: 63.20.Dj Phonon states and bands, normal modes, and phonon dispersion; 63.20.Kr Phonon-electron and phonon-phonon interactions.

Keywords: rare-gas solids, phonon spectrum, electron-phonon interaction, high pressure.

\section{Introduction}

The advent of new methods for studying materials has opened up new opportunities for the experimental investigation of phonon spectra under high pressures [1]. Investigations into the behavior of phonon frequencies under pressure provide valuable information on the structural instability, the mechanisms of phase transitions, and the interatomic interactions in the crystals. With knowledge of the phonon frequencies, one can easily calculate the thermodynamic properties of materials at high pressures.

The simplicity of rare-gas solids (RGSs) makes them particularly attractive for detailed studying lattice dynamics of solid $\mathrm{Ne}, \mathrm{Ar}, \mathrm{Kr}$ and $\mathrm{Xe}$ under pressure. It is known that the pressures required to investigate physical phenomena in cryocrystals are higher than those necessary for other materials $[1,2]$. This imposes a number of stringent requirements on the methods used for the phonon spectrum calculations. Therefore, it is necessary to choose computational methods such that they 1) do not contain fitting parameters (at least, in the short-range part of the interatomic potential); 2) do not operate with approximations for the crystal potential, which are poorly controllable with varying in pressure; 3) use function basis sets that are appropriate at any compression (includ- ing metallization pressures); 4) do not assume smallness of the overlap integrals of localized basis orbitals; and 5) allow for the inclusion of nonadiabatic effects (electron-phonon interactions).

In the articles [3-6] first-principles calculations for crystalline $\mathrm{Ne}, \mathrm{Ar}, \mathrm{Kr}$, and $\mathrm{Xe}$ have been performed to investigate phase relations, electronic structure, and vibrational properties of RGSs under pressure. These calculations are based on the density functional theory (DFT) [7] and the local density approximation (LDA) for the exchange-correlation potential [8] which should be recognized as a suitable method for the purpose of this study. However, LDA is known to fail to describe weakly bonded systems such as the van der Waals solids [9]. Yet, it is expected that a compression-related increase of the charge density in the internuclear region will be a validating argument for this approximation [10]. The authors of Ref. 6 «report on the first ab initio investigation of the lattice dynamics of fcc Xe. Not only is such an investigation useful for obtaining thermodynamic properties of xenon per se, but also serves to gauge the performance of density-functional and pseudopotential techniques for this class of materials».

However, in our opinion, direct consistent calculation of the dynamic-matrix elements is the most efficient technique, because, in this case, all the approximations 
used in the model perform at their best. In this respect, the development of methods appropriate for calculating the phonon frequencies at high pressures is an important problem.

In this paper, the lattice dynamics in RGSs has been investigated from first principles over a wide range of pressures with due regard for nonadiabatic effects. Analysis of these effects is of the utmost importance in describing the behavior of matter at pressures $p \neq 0$, when the adiabaticity parameter is not small and the electron and phonon spectra can overlap.

These calculations are based on the Tolpygo model and its variants [11-14]. In contrast to the standard approach, which is based on Green's functions (see, for example, [15]), the Tolpygo theory makes it possible to investigate quantitatively the electron-phonon interaction in crystals with a high binding energy over a wide range of pressures, including those corresponding to an insulator-metal transition.

\section{Interatomic potential and the parameters of electron-phonon interaction in compressed cryocrystals}

The dynamical theory of cryocrystal lattices with allowances for the deformation of electron shells in atoms (the Tolpygo model) was developed in $[12,13]$.

The crystal energy has the form

$$
E=\sum_{l}\left\{\left(\mathbf{P}^{l}\right)^{2} /(2 \alpha)+\beta^{l} \mathbf{P}^{l}+\frac{1}{2} \sum_{l \neq l^{\prime}} \kappa\left(\mathbf{P}^{l}, \mathbf{P}^{l^{\prime}}\right)+E^{(1)}+E^{(2)}\right\} .
$$

Here, $\mathbf{P}^{l}$ is the dipole moment of the atom at site $l$ induced by moving nuclei (higher multiple moments are neglected). The term $\beta^{l} \mathbf{P}^{l}$ is the exchange dipole interaction, and the third term is the dipole-dipole interaction.

The first three terms, which are associated with fluctuating deformations of the electron shells, are responsible for nonadiabatic effects (electron-phonon interaction). The last two terms in Eq. (1) are the energies of shortrange repulsion of atoms and of the long-range attraction due to many-particle effects, respectively [16,17]. They are expressed in terms of pairwise interaction potentials between the atoms

$$
\begin{gathered}
E^{(1)}=T_{e}+E_{s r} ; \quad E_{s r}=\frac{1}{2} \sum_{l^{\prime} \neq l} V_{s r}\left(R^{l l^{\prime}}\right), \\
E^{(2)}=\frac{1}{2} \sum_{l^{\prime} \neq l} V_{l r}\left(R^{l l^{\prime}}\right) .
\end{gathered}
$$

The term $E^{(2)}$ describes the attraction between the atoms; it is not allowed for the one-electron (Hartree-Fock) approximation and corresponds to virtual tran- sitions of two electrons from states $v_{1}$ and $v_{2}$ of the valence band to states $c_{1}$ and $c_{2}$ of the empty conduction band under the action of the electron-electron interaction operator. In the particular case of atomic shells that do not overlap (at large interatomic spacings), the energy $E^{(2)}$ reduces to the well-known van der Waals energy. The attraction energy between two atoms was calculated in [17] and can be written in the form

$$
\begin{gathered}
V_{l r}(R)=-\frac{C}{R^{6}}[1-f(x)] ; \quad f(x)=A \exp [-\beta(x-1)] ; \\
x=R / R_{0},
\end{gathered}
$$

where $C$ is van der Waals constant, $f(x)$ is a function associated with atomic electron shells overlap, $R$ is the nearest-neighbor distance in the compressed crystal and $R_{0}$ is the spacing between nearest neighbors in the crystal without compression; $A$ and $\beta$ are parameters (see [17]).

The short-range repulsion $V_{s r}(R)$ in Eq. (1) is calculated without recourse to variational of fitting parameters. This is of fundamental importance because the potential $V_{s r}(\mathbf{r})$ plays a decisive role in the calculation of the atomic properties of the compressed crystals. In [18], the potential $V_{s r}(\mathbf{r})$ was calculated from first principles at the Hartree-Fock level using the basis set of exactly orthogonalized atomic orbitals and the Abarenkov-Antonova cluster expansion [19]. In this case, the potential $V_{s r}(\mathbf{r})$ accounts for the contributions from higher order terms in the overlap integral of atomic orbitals $S$.

The short-range potential $V_{s r}(\mathbf{r})$ in the pair approximation can be represented in the form

$$
\begin{gathered}
V_{s r}\left(R^{l l^{\prime}}\right)=\left\langle 00\left|H_{l l^{\prime}}^{s r}\right| 00\right\rangle= \\
=2 \sum_{\alpha \beta}\left\{4 P_{\alpha \beta}^{l l^{\prime}}\left[-\left\langle l^{\prime} \beta\left|V^{l^{\prime}}\right| l \alpha\right\rangle+\sum\left\langle l^{\prime} \gamma, l^{\prime} \beta\left|V_{C}\right| l^{\prime} \gamma, l \alpha\right\rangle\right]+\right. \\
\left.+\sum_{\gamma}\left(P_{\beta \alpha}^{l l{ }^{\prime}} P_{\gamma \alpha}^{l l^{\prime}}-2 P_{\alpha \beta}^{l l}\right)\left(2\left\langle l^{\prime} \beta\left|V^{l}\right| l^{\prime} \gamma\right\rangle-\sum_{\delta}\left\langle l \delta, l^{\prime} \beta\left|V_{C}\right| l^{\prime} \gamma, l \delta\right\rangle\right)\right\} .
\end{gathered}
$$

Here, $V^{l}$ is the potential of neutral atom $l, V_{C}=1 /\left|\mathbf{r}-\mathbf{r}^{\prime}\right|$,

$$
\begin{gathered}
\left\langle l^{\prime} \gamma, l^{\prime} \beta\left|V_{C}\right| l^{\prime} \gamma, l \alpha\right\rangle= \\
=\int\left[\left(\varphi_{\gamma}^{l^{\prime}}(\mathbf{r}) \varphi_{\beta}^{l^{\prime}}\left(\mathbf{r}^{\prime}\right) \varphi_{\gamma}^{l^{\prime}}\left(\mathbf{r}^{\prime}\right) \varphi_{\alpha}^{l}(\mathbf{r})\right) /\left|\mathbf{r}-\mathbf{r}^{\prime}\right|\right] d \tau d \tau^{\prime},
\end{gathered}
$$

and the Greek indices denote the Cartesian components. Expression (5) transforms into the known relationship for the pair potential originally obtained in our earlier work [20] in the limit $S<<1$, i.e., with the use of the formulas

$$
P_{\alpha \beta}^{l l^{\prime}}=\frac{1}{2} S_{\alpha \beta}^{l l^{\prime}}+O\left(S^{2}\right), \quad P_{\alpha \beta}^{l l}=-\frac{3}{8} \sum_{m \alpha} S_{\gamma \alpha}^{l m} S_{\beta \alpha}^{l m}+O\left(S^{4}\right),
$$


where $S_{\alpha \beta}^{l l^{\prime}}$ is the overlap integral between two atomic orbitals centered at different sites:

$$
S_{\alpha \beta}^{l l^{\prime}}=\int \varphi_{\alpha}^{*}(\mathbf{r}-\mathbf{l}) \varphi_{\beta}\left(\mathbf{r}-\mathbf{l}^{\prime}\right) d \mathbf{r} .
$$

Compression, like any action that does not change the structure of the electron wave functions of an atom, affects the properties of the crystal only due to a change in the distances $\mathbf{l}-\mathbf{m}$ and the overlap of the atomic orbitals. In this sense, relations (7) for the overlap integrals $S_{\alpha \beta}^{l m}$ are the sole controlling parameters of the theory [21]. The set of overlap integrals $S_{\alpha \beta}^{l m}$ uniquely determines all the properties and the adiabatic potential (through the matrix $P(6)$ ). Therefore, the problem of calculating the properties of insulators is divided into two stages: (i) calculation of the set of overlap integrals $S_{\alpha \beta}^{l m}$ and (ii) calculation of the spectra and the thermodynamic and kinetic characteristics using at the known overlap integrals $S_{\alpha \beta}^{\operatorname{lm}}$.

\section{Phonons and electron-phonon interaction in RGSs under high pressure}

The equations of motion for RGSs were obtained in the harmonic approximation in [12]. Belogolovskii et al. [13] analyzed these equations and derived analytical expressions for the squares of the phonon frequencies as applied to the symmetric directions of the wave vector $\mathbf{K}$. These expressions for the $[00 \xi]$ direction are as follows:

$$
\begin{gathered}
\Omega_{L}^{2}=2(H+G)\left(1-\cos k_{z}\right)-\frac{(2 h+2 g)^{2}}{A^{-1}-\varphi_{z z}}\left(1-\cos k_{z}\right)^{2}+ \\
+(F+E) 2 \sin ^{2} k_{z}+B \chi_{z z} ; \\
\Omega_{T}^{2}=(G+2 H)\left(1-\cos k_{z}\right)-\frac{(2 h+g)^{2}}{A^{-1}-\varphi_{x x}}\left(1-\cos k_{z}\right)^{2}+ \\
+2 F \sin ^{2} k_{z}+B \chi_{x x},
\end{gathered}
$$

where $\mathbf{k}=a \mathbf{K}$ ( $a$ is half of the cube edge) and $\xi_{i}=k_{i} / \pi$. Similar relationships are available for the $[\xi \xi \xi]$ and $[\xi \xi 0]$ directions.

The tensors $\varphi_{i j}$ and $\chi_{i j}$ are lattice sums which depend on the wave vector $\mathbf{K}$ to be found in [22] and [13], respectively. In Eqs. (8) and (9), $A$ is the atomic polarizability divided by $a^{3}$ and related to the permittivity by the Clausius-Mossotti equation; $G, H, E$, and $F$ are the force parameters of the short-range interaction between the nearest and next-nearest neighbors, respectively ( $H$ and $F$ are the transverse elastic coefficients); $B$ is the Van der Waals constant divided by $(1 / 6) e^{2} a^{5}$; and $g$ and $h$ are the parameters of the electron-ion interaction.
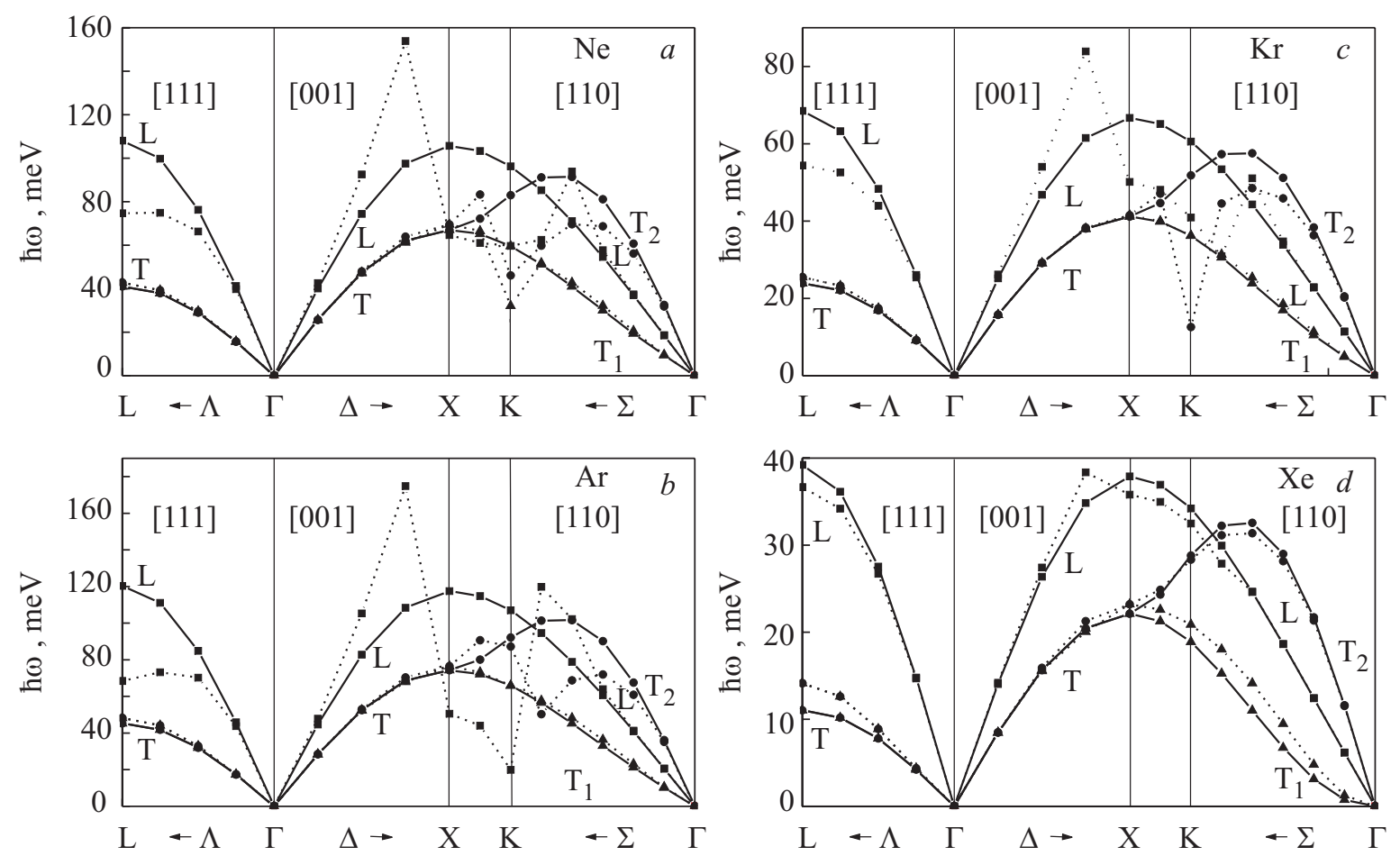

Fig. 1. Phonon dispersion curves for $\mathrm{Ne}(a), \operatorname{Ar}(b), \operatorname{Kr}(c)$, and $\mathrm{Xe}(d)$ crystals along high-symmetry directions of the wave vector $\mathbf{k}$ for compression ratios $\Delta V / V_{0}=0.7$. Solid curves indicate the transverse $T\left(T_{1}, T_{2}\right)$ and longitudinal $L$-branches along the $\Lambda, \Delta$ and $\Sigma$ directions as calculated within the $M 3$ model. Dashed curves represent the transverse $T\left(T_{1}, T_{2}\right)$ and longitudinal $L$ - branches calculated within the $M 3 a$ model. 
The relationships for the parameters $G, H, E$, and $F$, describing the repulsion, their calculated values, and the van der Waals parameters of RGSs for compressions $\Delta V / V_{0}$ in the range from 0 to 0.8 (where $V_{0}$ and $V$ are the unit cell volumes at $p=0$ and $p \neq 0$, respectively) are given in $[18,21,23]$. The parameters of the electron-phonon interaction $g, h$ were determined in $[24,25]$.

Let us now analyze the phonon frequencies of the neon, argon, krypton, and xenon crystals. As in [25], the simplest model $M 2$ corresponds to the nearest-neighbor approximation $(E=F=0)$ without account for the nonadiabatic contributions $(g=h=0)$ in which the potential $V_{s r}(1)$ is calculated in the $S^{2}$ approximation. The $M 3$ model additionally includes the next-to-nearest neighbors. The $M 3 a$ model takes into account the nearest and next-nearest neighbors and the nonadiabatic contributions $\left(V_{S r} \sim S^{2}\right)$. Figure 1 schematically depicts the phonon dispersion curves for the neon, argon, krypton, and xenon crystals at a compression of 0.7 .

We showed in $[24,25]$ that, as the compression ratio increases to below 0.6 , the frequencies $\hbar \omega_{\lambda}(\mathbf{k})$ increase by approximately one order of magnitude but the phonon dispersion curves remain smooth; by contrast, the electronic spectra for the neon crystal at a compressions above 0.6 exhibit distortions and «humps». In the phonon spectrum, as it can be seen from Fig. 1, the distortion is clearly pronounced in the longitudinal $L$ - and transverse $T_{2}$-modes along the $\Delta$ and $\Sigma$ directions for a compression of 0.7 when the nonadiabatic effects are taken into account (the calculation within the $M 3 a$ model). This implies a softening of these modes. The relative contribution $\gamma$ of these effects decreases in the sequence $\mathrm{Ar}, \mathrm{Kr}, \mathrm{Xe}$.

In our model, the compression 0.7 corresponds to a pressure of 136.6 GPa for Ne, 287.8 GPa for Ar, 212.7 $\mathrm{GPa}$ for $\mathrm{Kr}, 128.6 \mathrm{GPA}$ for Xe. For these crystals this is the region of insulator-metal phase transition, when the fordidden band gap vanishes $\left(E_{G} \rightarrow 0\right)[2,18]$.

\section{Discussion}

Unfortunately, there are few experimental works where phonon spectra at high pressures were studied $[1,26]$. In this respect, our calculations will be compared with the sole experiment available for argon. It was shown [26] that the phonon branches for a single crystal compressed in a diamond-anvil cell, can be in principle, accurately measured using inelastic $x$-ray scattering. The measurements were performed at pressures 3.1 and $20 \mathrm{GPa}$ and could be extended to Mbar pressures. However, the best results were obtained at $3.1 \mathrm{GPa}$.

The experimental [26] and theoretical phonon frequencies calculated in the $M 3 a$ model are presented in Fig. 2. The compression ratio is chosen to be equal 0.246 , which corresponds to the experimental lattice parameter

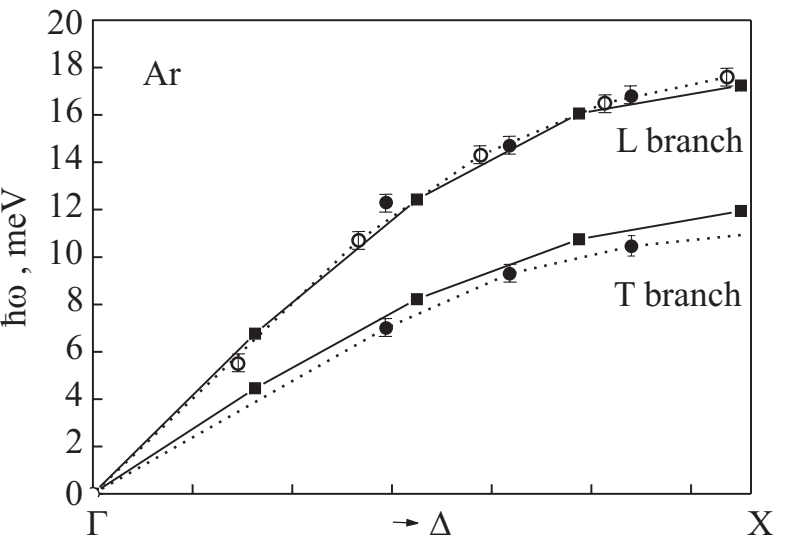

Fig. 2. Experimental and theoretical phonon frequencies of the Ar crystal along the $[00 \xi]$ direction at the compression ratio $\Delta V / V_{0}=0.246$. Solid curves with closed squares indicate the results of calculations within the $M 3 a$ model. The dotted line with open circles corresponds to the experimental data obtained for the first Brillouin zone [26]. The dotted line with closed circles represents the experimental data obtained for the second Brillouin zone [26].

(i.e., the cube edge $a_{\exp }=4.845 \AA, p=3.1 \mathrm{GPa}$ [26], $\left.a_{\text {theor }}=4.842 \AA, p=2.6 \mathrm{GPa}[18]\right)$. It can be seen from Fig. 2 that the calculations agree well with experiment. The calculations performed with an allowance for nonadiabatic contributions lead to the smallest relative error $\delta$. The $X$ point is especially characteristic: at this point, the inclusion of the electron-phonon interaction results in an almost ideal agreement between the longitudional frequencies $\omega_{L}^{\text {theor }}$ and $\omega_{L}^{\exp }$ (the relative error $\delta$ decreases from 2.9 to $0.9 \%$ ). The transverse branch is described somewhat worse.

Dewhurst et al. [16] used DFT to obtain phonon spectra of fcc xenon under pressure. They found that all phonon modes monotonically increase with pressure up to $100 \mathrm{GPa}$ beyond which the transverse acoustic modes at the $X$ - and $L$-points start to soften $\left(\hbar \omega_{T}(X)=\right.$ $=21.3 \mathrm{meV} ; 20.9 \mathrm{meV} ; 19.0 \mathrm{meV}$ and $\hbar \omega_{T}(L)=13.1 \mathrm{meV}$; $13.1 \mathrm{meV} ; 13.0 \mathrm{meV}$ at $p=100 \mathrm{GPa} ; 110 \mathrm{GPa} ; 120 \mathrm{GPa}$, respectively).

The values of the frequencies are approximately the same as ours (within $M 3 a, \hbar \omega_{T}(X)=23.2 \mathrm{meV}$ and $\hbar \omega_{T}(L)=14.1 \mathrm{meV}$ at $p=128 \mathrm{GPa}$, within the $M 3$ model $\hbar \omega_{T}(X, L)$ is somewhat lower, see Fig $\left.1, d\right)$. However, we have shown that at the $X$ - and $L$-points the longitudinal modes «soften» and the electron-phonon interaction contributes positively to the transverse modes. For longitudinal and transverse modes one can easily determine the sign of the contribution from the electron-phonon interaction $\left(\Omega_{\lambda}^{2}\right)^{*}$ into $\Omega_{\lambda}^{2}$ at the $X$-point. From Eqs. (6) and (7) for the direction of $k \|[00 \xi]$ we have: 


$$
\begin{aligned}
& \left(\Omega_{L}^{2}\right)^{*}=-\frac{(2 h+2 g)^{2}}{A^{-1}-\varphi_{z z}}\left(1-\cos k_{z}\right)^{2}, \\
& \left(\Omega_{T}^{2}\right)^{*}=-\frac{(2 h+g)^{2}}{A^{-1}-\varphi_{x x}}\left(1-\cos k_{z}\right)^{2} .
\end{aligned}
$$

For all RGSs, with $p>20 \mathrm{GPa}$, the polarizability $A>1.0$ and the sign of the contribution is determined by the sign of $\varphi_{\alpha \beta}$. At the $X$-point in crystals with fcc structure [22], $\varphi_{z z}<0, \varphi_{x x}>0$, and $\left|\varphi_{\alpha \beta}\right|>A^{-1}$, therefore $\left(\Omega_{L}^{2}\right)^{*}<0,\left(\Omega_{T}^{2}\right)^{*}>0$ at the $X$-point. Similarly, $\left(\Omega_{L}^{2}\right)^{*}<0,\left(\Omega_{T}^{2}\right)^{*}>0$ at the $L$-point.

Nonadiabatic effects are enhanced more clearly when electrons interact stronger with phonon branches, i.e. the $L$-modes along all high-symmetry directions and $T_{2}$-mode along the $\Sigma$ direction where $\hbar \omega_{T_{2}}>\hbar \omega_{L}$. This is also confirmed by the fact that in the sequence $\mathrm{Ne}$ to $\mathrm{Xe}$ series the relative contribution of the electron-phonon interaction $\gamma$ is the largest for $\operatorname{Ar}$ (see Fig.1, $a-d$ ). For example, at the $X$-point ( $L$-mode), for compression $\Delta V / V_{0}=0.7, \gamma=38.8 \%, 57 \%, 24.9 \%$ and $5.5 \%$ for $\mathrm{Ne}$, $\mathrm{Ar}, \mathrm{Kr}$ and $\mathrm{Xe}$, respectively. In our opinion, theory [16] lacks explanation of the mechanism and the extent of the phonon «softening», and this can be done by using the theory proposed here.

\section{Conclusions}

In this study, as in $[18,24,25,27]$, the adiabatic potential $E$ was constructed using a general approach. This approach as applied for rare-gases crystals in the sequence $\mathrm{Ne}-\mathrm{Xe}$ makes it possible to determine the most important interactions in these crystals, i.e., the structure of the interatomic potentials. For this purpose, we analyzed six models of interatomic interaction in rare-gas crystals [24,25]. It was demonstrated that the $M 2$ model for argon, krypton, and xenon and the model $M 4$ for neon are quite consistent; they are based on clear physical principles and well formulated approximations, and adequately describe the phonon frequencies at low pressures and temperatures. At high pressures, the models allowing for the electron-phonon interaction (i.e., the models $M 3 a$ and M5 for neon and the model $M 3 a$ for other rare-gas crystals) yield the best results.

In order to construct the potential for neon, it is necessary to take into account the pair terms of higher orders in $S$, whereas the potentials for other crystals can be constructed with the inclusion of the terms $\sim S^{2}$. This is explained by the fact that the short range potential $V_{s r}$ is a small difference between large quantities [20]:

$$
V_{s r}=V_{s r}^{+}+V_{s r}^{-} \text {. }
$$

Moreover, for argon, krypton, and xenon crystals, the short-range potential $V_{s r}$ amounts to $40-50 \%$ of the potential $V_{s r}^{+}$. However, the ratio $V_{s r} / V_{s r}^{+}$for neon is $20-25 \%$. Therefore, the terms of higher orders in $S$ for argon, krypton, and xenon are small corrections, whereas their contribution to the potential for neon is comparable to the terms $\sim S^{2}$.

Thus, the theory developed makes it possible to calculate the short-range repulsive potential for all rare gas crystals without recourse to variational or fitting procedures.

In our opinion, the $a b$ initio calculation of the shortrange repulsive potential is the main requirement for a theory that claims to adequately describe the properties of materials under pressure.

The calculations of the phonon frequencies for all RGSs made it possible to determine the contributions from the various interactions in these crystals. Neon is a typical representative of low $-Z$ materials. Apart from the quantum effects observed at $T=p=0$ [28], neon is characterized by effects that manifest themselves at high pressures, such as nonadiabatic effects and contributions from the terms of higher orders in $S$ to the potential $V_{s r}$. For other rare-gas cryocrystals, it suffices to use the approximation $V_{s r} \sim S^{2}$. The contribution from the electron-phonon interaction to the phonon frequencies is also large for Ar and is considerably smaller for the krypton and xenon crystals.

Note that in the classical case of metals the shift of the phonon frequency $\Delta \omega_{\lambda q}$ due to the electron-phonon interaction (see, for example, [15, p. 40]) is determined by the expression

$$
\hbar \Delta \omega_{\lambda_{\mathbf{q}}}=\sum_{\mathbf{k}}\left|M_{\mathbf{k q} \lambda}\right|^{2}\left|\frac{n_{\mathbf{k}}-n_{\mathbf{k}+\mathbf{q}}}{\varepsilon_{\mathbf{k}}-\varepsilon_{\mathbf{k}+\mathbf{q}}-\hbar \omega_{\lambda \mathbf{q}}}-\frac{n_{\mathbf{k}}-n_{\mathbf{k}+\mathbf{q}}}{\varepsilon_{\mathbf{k}}-\varepsilon_{\mathbf{k}+\mathbf{q}}}\right| .
$$

The second term describes the adiabatic contribution of electrons, to the phonon frequency, i.e., the contribution that is associated with the account of the electron energy in the equation for the diagonal ionic wave function and which is of the same order as the phonon frequency $\omega_{\lambda \mathbf{q}}$.

The phonon frequency is a very sensitive characteristic (in contrast to the thermodynamic properties, which are integral functions of frequency). By using this specific feature, it has become possible to determine the contribution from terms of higher orders in $S$ to the potential $V_{s r}$ even at low compressions more accurately than in the band-structure calculations of the neon crystal [2,21].

In conclusion, we note that in the adiabatic theory of perturbations in the adiabaticity parameter $\kappa^{2}[15]$ there are two types of terms of different physical origins, namely, the electron-phonon and anharmonic (phonon-phonon) corrections to the energy of the electronic subsystem. The quantitative investigations into the nonadiabatic effects at high pressures allowed us to draw the 
conclusion that the structural instability and the generation of a soft mode in crystals with a high binding energy are caused by the electron-phonon interaction.

1. M. Krisch, J. Raman Spectrosc. 34, 628 (2003).

2. E.V. Zarochentsev and E.P. Troitskaya, Fiz. Tverd. Tela (St. Petersburg) 44, 1309 (2002) [Phys. Solid State 44, 1370 (2002)].

3. I. Kwon, L.A. Collins, J.D. Kress, and N. Troullier, Phys. Rev. B52, 15165 (1995).

4. W.A. Caldwell, J.H. Nguyen, B.G. Pfrommer, F. Mauri, S.G. Louie, and R. Jeanloz, Science 277, 930 (1997).

5. M. Springbord, J. Phys.: Condens. Matter 12, 9869 (2000).

6. J.K. Dewhurst, R. Ahuja, S. Li, and B. Johansson, Phys . Rev. Lett. 88, 075504 (2002).

7. P. Hohenberg and W. Kohn, Phys. Rev. 126, B864 (1964).

8. W. Kohn and L.J. Sham, Phys. Rev. 140, A1133 (1965).

9. W. Kohn, Y. Meir, and D.E. Makarov, Phys. Rev. Lett. 80, 4153 (1998).

10. T. Tsuchiya and K. Kawamura, J. Chem. Phys. 117, 5859 (2002).

11. K.B. Tolpygo, Zh. Eksp. Teor. Fiz. 20, 497 (1950).

12. K.B. Tolpygo and E.P. Troitskaya, Fiz. Tverd. Tela (Leningrad) 13, 1135 (1971) [Sov. Phys. Solid State 13, 939 (1971)].

13. M.A. Belogolovskii, K.B. Tolpygo, and E.P. Troitskaya, Fiz. Tverd. Tela (Leningrad) 13, 2109 (1971) [Sov. Phys. Solid State 13, 1765 (1971)].

14. E.P. Troitskaya, Doctoral Dissertation in Physics and Mathematics, Kiev (1987).

15. V.G. Bar'yakhtar, E.V. Zarochentsev, and E.P. Troitskaya, Theory of Adiabatic Potential and Atomic Properties of Simple Metals, Gordon and Breach, London (1999).

16. V.L. Dorman, E.V. Zarochentsev, and E.P. Troitskaya, Fiz. Tverd. Tela 23, 1581 (1981) [Sov. Phys. Solid State 23, 925 (1981)].
17. V.L. Dorman, E.V. Zarochentsev, and E.P. Troitskaya, Fiz. Nizk. Temp. 8, 94 (1982) [Sov. Low Temp. Phys. 8, 47 (1982)].

18. E.V. Zarochentsev and E.P. Troitskaya, Fiz. Tverd. Tela (St. Petersburg) 43, 1292 (2001) [Phys. Solid State 43, 1345 (2001)].

19. I.V. Abarenkov and I.M. Antonova, Fiz. Tverd. Tela (Leningrad) 20, 565 (1978) [Sov. Phys. Solid State 20, 326 (1978)].

20. K.B. Tolpygo and E.P. Troitskaya, Fiz. Tverd. Tela (Leningrad) 17, 102 (1975) [Sov. Phys. Solid State 17, 58 (1975)].

21. V.G. Bar'yakhtar, E.V. Zarochentsev, E.P. Troitskaya, and Yu.V. Eremeichenkova, Fiz. Tverd. Tela (St. Petersburg) 40, 1464 (1998) [Phys. Solid State 40, 1330 (1998)].

22. K.B. Tolpygo and I.G. Zaslavskaya, Ukr. Fiz. Zh. 1, 226 (1956).

23. E.V. Zarochentsev, E.P. Troitskaya, and V.V. Chabanenko, Fiz. Tekh. Vys. Davlenii 11, 7 (2001).

24. E.P. Troitskaya, V.V. Chabanenko, and E.E. Horbenko, Fiz. Tverd. Tela (St. Petersburg) 47, 1683 (2005) [Phys. Solid State 47, 1748 (2005)].

25. E.P. Troitskaya, Val.V. Chabanenko, and E.E. Horbenko, Fiz. Tverd. Tela (St. Petersburg) 48, 695 (2006) [Phys. Solid State 48, 741 (2006)].

26. F. Occelli, M. Krisch, P. Loubeyre, F. Sette, R.Le Toullec, C. Masciovecchio, and J.-P. Rueff, Phys. Rev. B63, 224306 (2001).

27. E.V. Zarochentsev, E.P. Troitskaya, and V.V. Chabanenko, Fiz. Tverd. Tela (St. Petersburg) 46, 245 (2004) [Phys. Solid State 46, 249 (2004)].

28. E.V. Zarochentsev, E.P. Troitskaya, and V.V. Chabanenko, Fiz. Tekh. Vys. Davlenii 13, 7 (2003). 